\title{
Eine Chance für die Schweizer Transplantationsmedizin
}

Franz F. Immer

PD Dr. med., Facharzt für Herzchirurgie FMH und CEO Swisstransplant, Mitglied FMH

In diesem Jahr ist die Schweiz Gastgeberland des Europäischen Organspendetags EODD. Am 9. September feiert Swisstransplant auf dem Bundesplatz in Bern, zusammen mit Spendern und Empfängern, mit dem BAG, der Politik und Persönlichkeiten des öffentlichen Lebens. Dieser Tag gibt uns die Möglichkeit, uns international mit Experten auf dem Gebiet der Organspende und der Transplantation auszutauschen und vor allem die Bevölkerung für das Thema zu sensibilisieren.

Zusammen mit einem Gastgeberland organisiert der Europarat seit 1996 jährlich den «European Day for Organ Donation and Transplantation» (EODD) zur Sensibilisierung der Öffentlichkeit. Dieser Tag ist Teil der gemeinsamen Anstrengungen der Europäischen Kommission, des Europarats und der Mitgliedstaaten, die Anzahl der Organspender zu erhöhen, die Transplantationsmedizin zu unterstützen und somit die Qualität und Sicherheit von Transplantationen weiter zu verbessern. 2017 ist die Schweiz Gastgeberin des EODD. Parallel dazu findet in Genf ein Festakt zum «World Day of Organ Donation and Transplantation» (WDODT) statt. Und auch der Nationale Tag der Organspende fällt auf dasselbe Datum. Damit werden erstmals in der Geschichte dieser Gedenktage alle drei Anlässe am selben Tag im selben Land stattfinden.

\section{Pionierrolle der Schweiz}

Dass die Schweiz 2017 Gastgeberin des EODDs ist, versteht Swisstransplant auch als Würdigung der Rolle der Schweiz in der Geschichte der Transplantationsmedizin. Diese nahm 1883 ihren Anfang, als der Berner Chirurg und spätere Nobelpreisträger Theodor Kocher (1841-1917) einem jungen Mann, dem die Schilddrüse entfernt worden war, menschliches Schilddrüsen-

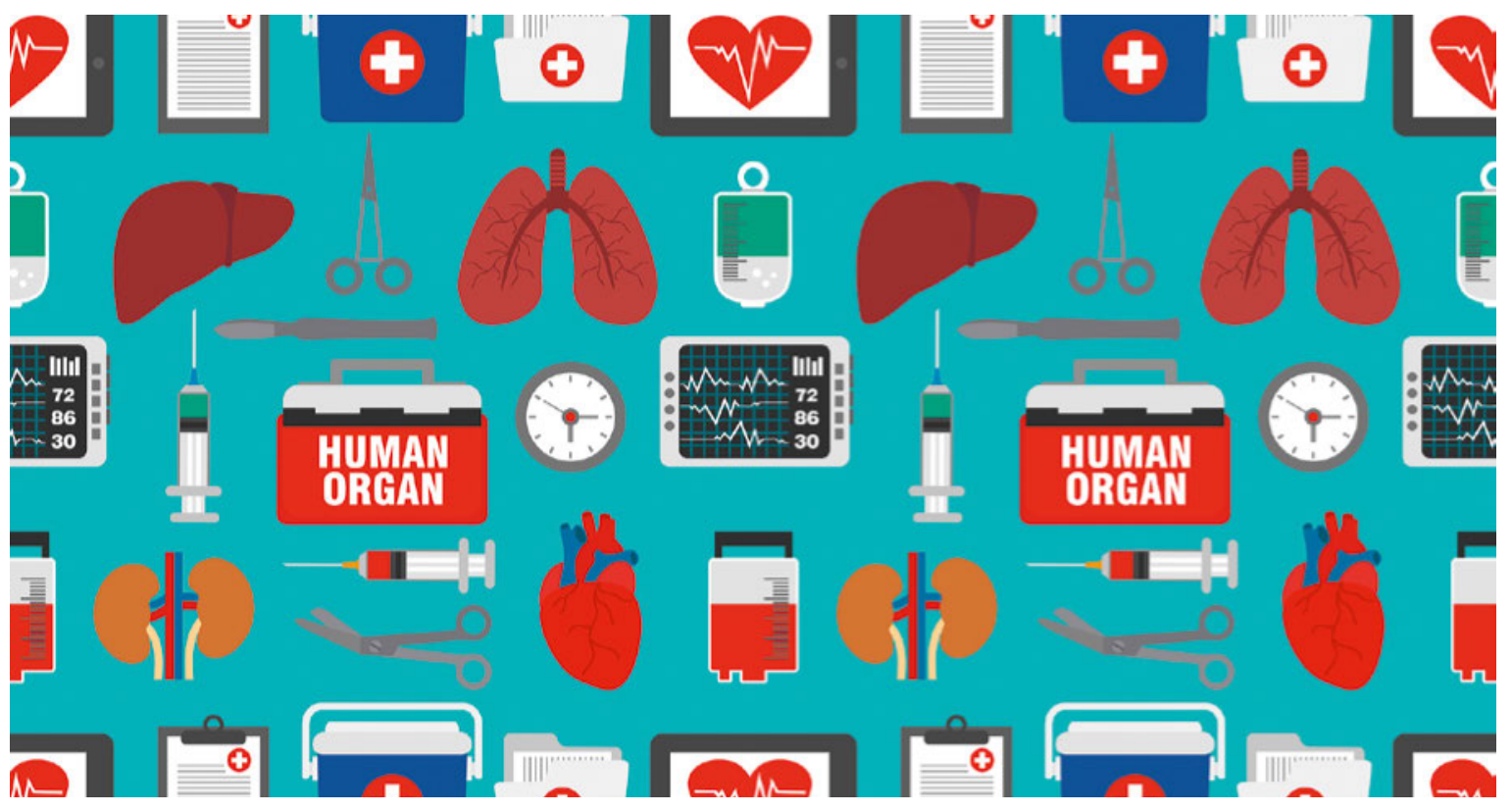

Am 9. September soll über Organspende gesprochen und die breite Öffentlichkeit sensibilisiert werden. 
gewebe unter die Haut und in die Bauchhöhle verpflanzte. Das war damals ein grosser Durchbruch und gilt als Beginn der Transplantationstherapie. Ein weiterer Meilenstein war die Entdeckung von Ciclosporin durch Hartmann Stähelin und Jean-François Borel bei Sandoz in Basel Anfang der 1970er-Jahre. Daraus wurden die ersten Immunsuppressiva entwickelt, die die Abstossung transplantierter Organe verhindern. Seitdem hat die Schweiz immer wieder ihren Beitrag zur steten Weiterentwicklung der Transplantationsmedizin geleistet. Es ist uns eine Ehre, Gastgeberin des EODD 2017 zu sein und wir freuen uns auf den Besuch und regen Austausch mit vielen europäischen Spezialisten auf dem Gebiet von Organspende und Transplantation.

\section{Die Idee des EODD}

Die Idee hinter dem Tag ist es, jedes Jahr einen anderen Mitgliedsstaat dabei zu unterstützen, das öffentliche Bewusstsein zu fördern und die Debatte über Organspende und Transplantation anzuregen. Der Mangel an transplantierbaren Organen führt nicht nur in der Schweiz, sondern auch europaweit zu einer längeren Warteliste. Zudem nimmt der Bedarf an Organen laufend $\mathrm{zu}$. Mit den richtigen Informationen möchte Swisstransplant dafür sorgen, dass jeder und jede sich zum Thema Organspende Gedanken macht, sich entscheidet und diesen Entscheid seinen bzw. ihren Angehörigen mitteilt. Auch Spitäler und einzelne Fachpersonen werden uns an diesem Tag unterstützen, mit dem Ziel, mehr potentielle Spender zu mobilisieren. Der Gedenktag ist auch eine Gelegenheit, alle Spender und ihre Familien zu würdigen und Organspende- und Transplantationsfachleuten in ganz Europa zu danken, deren Arbeit hilft, Leben $\mathrm{zu}$ retten und die Lebensqualität vieler Menschen zu verbessern.

\section{Gesichter und Geschichten}

Swisstransplant hat den EODD zum Anlass genommen, mit einer Social-Media-Kampagne das Bewusstsein für die Organspende zu wecken, Vorurteilen zu begegnen und falsche Meinungen, die es dazu gibt, zu berichtigen. In sechs zwei- bis dreiminütigen Minidokus werden Persönlichkeiten der Organspende und der Transplantation porträtiert: die ärztlichen Leiter der Schweizer Spendenetzwerke, Spendewillige, eine Transplantationschirurgin, Organempfänger und Angehörige von Spendern. Da ist das ungleiche Freundespaar Matthias und Gianni: sie haben sich über ihr gemeinsames Schicksal, Organempfänger zu sein, kennengelernt und spielen heute zusammen Boule, um sich auf die Meisterschaft für Transplantierte vorzubereiten. Da ist Michelle, die so voller Lebensenergie und Tatendrang ist - niemand würde vermuten, dass sie herztransplantiert ist. Oder Baavalan, der als Kind lebertransplantiert wurde, heute Medizin studiert und sich in Genf bei der Ärztin, die ihn damals operiert hat, für ein Praktikum bewirbt. Da ist Sébastian, der Fahrlehrer und Rennfahrer, den sein gefährliches Hobby und ein Krankheitsfall in der Familie dazu bewogen haben, ja zur Organspende zu sagen. Da ist Barbara, die ihren Mann verloren hat und in dieser schwierigen Situation die Entscheidung zur Organspende selber fällen musste, weil das Thema in der Familie vorher nicht besprochen wurde.

Diese bewegenden Geschichten zeigen, wie wichtig es ist, über Organspende zu reden, sich zu entscheiden und seine Angehörigen dadurch zu entlasten. Sie finden diese Filme und weitere Informationen zum EODD auf www.eodd2017.ch

Wir freuen uns auf Ihren Besuch auf dem Berner Bundesplatz!

\section{Bildnachweis}

(c) Dicraftsman | Dreamstime.com 